\title{
Impact of Intraventricular Hemorrhage on Classification of Hematoma Expansion and Development of Radiomics Prediction Models
}

Te-Chang Wu ( $\sim$ porthoswu@yahoo.com.tw)

Chi-Mei Medical Center

Yan-Lin Liu

University of California, Irvine

Jeon-Hor Chen

University of California, Irvine

Yang Zhang

University of California, Irvine

Tai-Yuan Chen

Chi-Mei Medical Center

Ching-Chung Ko

Chi-Mei Medical Center

Min-Ying Su

University of California, Irvine

\section{Research Article}

Keywords: intracerebral hemorrhage, intraventricular hemorrhage, hematoma expansion, radiomics

Posted Date: October 18th, 2021

DOl: https://doi.org/10.21203/rs.3.rs-963961/v1

License: (9) (i) This work is licensed under a Creative Commons Attribution 4.0 International License. Read Full License 


\section{Abstract}

Background: To investigate the impact of intraventricular hemorrhage (IVH) on the classification of hematoma expansion $(\mathrm{HE})$, and the development of radiomics models using features extracted from the baseline hematoma to predict $\mathrm{HE}$.

Methods: Eighty-four patients with baseline and follow-up non-contrast CT within 4 24 hours were included. The intraparenchymal hemorrhage (IPH) and IVH were separately outlined by an experienced neuroradiologist. HE was defined as an absolute hematoma growth $>6 \mathrm{~mL}$ or percentage growth $>33 \%$. $\mathrm{HE}$ was determined based on two criteria, using IPH alone $\left(\mathrm{HE}_{\mathrm{P}}\right)$ or IPH+IVH $\left(\mathrm{HE}_{\mathrm{P}+\mathrm{V}}\right)$. The radiomics analysis was performed by using PyRadiomics to extract features, followed by random forest algorithm to select features, and lastly the decision tree to build classification models.

Results: The classification of expansion showed $37(44 \%) \mathrm{HE}_{\mathrm{P}}$ and 47 (56\%) non-HE $\mathrm{E}_{\mathrm{P}}$ based on IPH alone, and similar results of $38(45 \%) \mathrm{HE}_{\mathrm{P}+\mathrm{V}}$ and $46(55 \%)$ non- $\mathrm{HE}_{\mathrm{P}+\mathrm{V}}$ based on IPH+IVH. The majority, $>94 \%$ of HE patients, had a poor outcome (death or $\mathrm{mRS}>3$ at discharge). Three radiomics analysis (RA) models were built. The first model using baseline IPH to predict $H E_{P}\left(R A_{P-P}\right)$ showed an accuracy of $80 \%$ but loss of correlation with the clinical outcome; the second model using IPH+IVH to predict $\mathrm{HE}_{\mathrm{P}}\left(\mathrm{RA} \mathrm{A}_{\mathrm{PV}-\mathrm{V}}\right)$ had a slightly higher accuracy of $81 \%$ and resumed the poor outcome association with $\mathrm{HE}$; and the third model using IPH+IVH to predict $\mathrm{HE}_{\mathrm{P}+\mathrm{V}}\left(\mathrm{RA} \mathrm{PV}_{\mathrm{PVV}}\right)$ had the highest accuracy of $86 \%$ with preserved clinical outcome correlation of HE. The sensitivity, specificity, and accuracy of three decision trees (RAP-P, RA PV-p, $\mathrm{RA}_{\mathrm{PV} \text {-PV }}$ ) were $0.8 / 0.68 / 0.89 ; 0.81 / 0.92 / 0.72$ and $0.86 / 0.82 / 0.89$, respectively.

Conclusions: The proposed radiomics approach with additional IVH information could be used to classify the expansion status highly associated with the clinical outcome and provide a robust tool for the enrollment of high-risk ICH cases in the anti-expansion trials.

\section{Introduction}

Spontaneous intracerebral hemorrhage (sICH) accounts for about 7-15\% of all strokes and carries a mortality rate of about $40 \%$, with half of the fatalities occurring within the first two days after an ictus (1$3)$. The high rate of early neurological deterioration after $\mathrm{sICH}$ is in part related to active bleeding that may proceed for hours after symptom onset(4). Among patients undergoing head CT scans within 3 hours of sICH onset, $28-38 \%$ have hematoma expansion (HE) on follow-up CT scans, with volume greater than one third compared with the hematoma volume on original CT scans $(3,4)$. Furthermore, HE had been proved to be an independent predictor of clinical deterioration and poor outcome $(3,5-7)$.

Several radiological predictors on the baseline non-contrast CT (NCCT) for HE had been proposed, such as hematoma volume, shape, hypodensities, density heterogeneity...etc(8-13). The pattern of heterogeneity can be analyzed using the texture features extracted by the radiomics approach, which has been shown capable of capturing various agnostic features to aid-in HE prediction(14-19). The 
radiomics features could be further combined with clinical $(19)$ and radiological variables $(16,17)$ to improve HE prediction accuracy.

Except for hematoma expansion in the brain parenchyma, the presence of intraventricular hemorrhage (IVH) at the baseline CT scan has been shown associated with mortality in patients with $\operatorname{siCH}(3,20,21)$. It was quoted as one risk factor in the ICH score(20), a clinical grading scale for risk stratification of sICH. Studies have reported that 30 to $50 \%$ of sICH patients experienced additional IVH(21). Recently, similar to $\mathrm{HE}$, IVH expansion at follow-up CT has also been identified as a strong predictor of poor clinical outcome(22). It was shown that the inclusion of IVH expansion into the definition of HE can improve the overall prediction accuracy of the 90-day outcome(23). Nevertheless, the IVH information was usually ignored in the conventional radiomics models using texture analysis(14-19).

In this retrospective study, we aimed to investigate the impact of IVH on the radiomic analysis for $\mathrm{HE}$ prediction as compared to the conventional approach based on intraparenchymal hemorrhage (IPH). Three different radiomics analyses were performed: 1) using IPH to predict expansion defined based on $\mathrm{IPH}$, noted as $\mathrm{RA}_{\mathrm{p}-\mathrm{p}}$; 2 ) using IPH+IVH to predict the traditional expansion defined based on IPH, noted as $\left.\mathrm{RA}_{\mathrm{PV}-\mathrm{P}} ; 3\right)$ using IPH+IVH to predict expansion defined based on IPH+IVH, noted as RAPV-PV. The prediction performance was compared.

\section{Material And Methods}

\section{Study population}

This study was approved by the Institutional Review Board of our hospital. The requirement to obtain informed consent was waived due to its retrospective nature. We reviewed the sICH database from the picture archiving and communication system (PACS) to identify the patients who underwent a baseline and a F/U NCCT within 4-24 hours from January 2014 to June 2018. In total, 119 patients were identified. The exclusion criteria included: 1 ) the co-existence of vascular lesions and brain tumor diagnosed during the same admission ( $\mathrm{N}=10)$; 2 ) pediatric patients <18 years old $(\mathrm{N}=2)$; 3 ) patients who underwent brain surgery before follow-up CT ( $\mathrm{N}=19)$; 4) patients with primary IVH and minimal IPH ( $\mathrm{N}=4)$. Thus, a total of 84 patients (61males, 23 females; mean age 60.1 12.4 years; range 34-94 years) were included in the analysis. Clinical information, including blood pressure (SBP>180 or $<180 \mathrm{mmHg}$; DBP $>100$ or $<100 \mathrm{mmHg}$ )(24), bleeding diathesis (INR $>1.5$, aPTT ratio $>1.5$ or platelet count $<1 \times 10^{5} / \mathrm{ml}$ ) $(25)$, Glasgow Coma Scale (GCS)(20) at admission (13 15 or $<13$ ), and mRS(26) at discharge ( $\leq 3$ or $>3$ ) were collected. The in-hospital mortality and modified Rankin Scale (mRS) at discharge, were used as the outcome.

\section{CT Imaging Protocol}

The brain CT was acquired using our standard protocol on a 64-slice CT (Definition AS; Siemens Medical Solutions, Forchheim, Germany). The scanning range was from the skull base to the cranial vertex with the following parameters: $120 \mathrm{kVp}, 380 \mathrm{mAs}$, and slice thickness/spacing of 4.8/4.8 mm. 


\section{Manual Hematoma Segmentation and HE definition}

The segmentation of the ICH region of interest (ROI) was performed manually, using Image $\mathrm{J}$ (National Institutes of Health, Bethesda, MD). The ROI drawing for baseline and F/U CT of each patient was done in the same seating by a neuroradiologist(TCW with 14 years of experience). The IPH and IVH were outlined separately, to form two datasets: $\mathrm{ICH}_{\mathrm{P}}$ containing the ROIs of IPH; and $\mathrm{ICH}_{\mathrm{P}+\mathrm{V}}$ containing the ROIs of IPH and IVH. Based on the hematoma volumetric change between baseline and F/U CT studies, HE was defined as an absolute hematoma growth $>6 \mathrm{~mL}$ or relative growth of $>33 \%$ from the baseline $\mathrm{ICH}(5,27)$. For $\mathrm{ICH}_{\mathrm{P}+\mathrm{V}}$, there has no consensus definition of expansion, so the same criteria were applied. After the $\mathrm{HE}$ status was defined, the baseline ROIs of $\mathrm{ICH}_{\mathrm{P}}$ and $\mathrm{ICH}_{\mathrm{P}+\mathrm{V}}$ were used to extract radiomics features, followed by feature selection and model building to predict HE.

\section{Feature Extraction}

The radiomics analysis (RA) procedures are illustrated in Figure 1.

For the $\mathrm{ICH}_{\mathrm{P}}$ or $\mathrm{ICH}_{\mathrm{P}+\mathrm{V}}$ in one patient, all segmented ROls on different slices were combined to form a 3D lesion mask, and the linear interpolation was utilized to convert the hematoma ROI to be isotropic. Then, a total of 107 features were calculated using the PyRadiomics, including 14 shape, 18 first-order, 24 Gray Level Co-occurrence Matrix texture, 14 Gray Level Dependence Matrix texture, 16 Gray Level Run Length Matrix texture, 16 Gray Level Size Zone Matrix texture, and 5 Neighboring Gray Tone Difference Matrix texture. To select robust features, two separate lesion ROI drawing was performed in 30 randomly selected cases. The extracted features from two ROls of the same lesion were correlated to calculate the intraclass correlation coefficient (ICC). Only features with ICC $>0.8$ were considered in the subsequent analysis to build models.

\section{Feature Selection and Decision Tree Model}

The extracted features from baseline $\mathrm{ICH}_{\mathrm{P}}$ or $\mathrm{ICH}_{\mathrm{P}+\mathrm{V}}$ were used to build radiomics models to predict expansion, using two definitions for $\mathrm{HE}_{\mathrm{P}}$ and $\mathrm{HE}_{\mathrm{P}+\mathrm{V}}$. Three different analyses were performed: 1) using $\mathrm{IPH}$ to predict $\mathrm{HE}_{\mathrm{P}}$, noted as $\mathrm{RA}_{\mathrm{P}-\mathrm{P}} ; 2$ ) using IPH+IVH to predict $\mathrm{HE}_{\mathrm{P}}$, noted as $\mathrm{RA} A_{\mathrm{PV}-\mathrm{P}} ; 3$ ) using IPH+IVH to predict $\mathrm{HE}_{\mathrm{P}+\mathrm{V}}$, noted as $\mathrm{RA} A_{\mathrm{PV}-\mathrm{PV}}$. In this study, we applied the random forest algorithm to estimate the feature importance as the selection criteria, by permutation of out-of-bag feature observation. The bootstrap-aggregated decision trees(28) were used to evaluate the importance of these features in differentiating patients with and without $\mathrm{HE}$. The significance of one selected feature could be assessed according to the decreased accuracy after this specified feature was removed. All features were sorted based on their importance, and then the different number of features starting from the top 1, 2, 3... was used to test their classification performance by using the binary decision tree. The split of the tree was based on the improvement of the cross-entropy. For each node, the cross-entropy of the classification results was calculated using the following formula: 


$$
\text { CrossEntropy }=-\sum_{i=1}^{k} p_{i} \log \left(p_{i}\right)
$$

In which $\mathrm{k}$ is the number of classes and pi is the proportion of cases belonging to class $\mathrm{i}$. For all of the parent and child nodes, the splitting of the nodes was determined by the threshold minimizing the crossentropy. The random forest and decision tree analysis were implemented using MATLAB 2019b.

\section{Statistical Analysis}

Statistical analyses of the clinical parameters were performed using the SPSS for Windows (V.24.0, IBM, Chicago, Illinois, USA). Discrete variables were presented as counts(n) and percentages(\%), and continuous variables were presented as medians and interquartile ranges (IQR). Chi-square test and student t-test were performed for categorical and continuous data respectively. $P$ values $<0.05$ was considered statistically significant.

\section{Results}

\section{Hematoma Expansion Status Defined Using IPH $\left(\mathrm{HE}_{\mathrm{P}}\right)$}

The baseline $\mathrm{ICH}$ volume, change of $\mathrm{ICH}$ volume at $\mathrm{F} / \mathrm{U}$, and short-term outcome of all 84 patients are summarized in Table 1. 
Table 1

The initial hematoma volume, change in follow-up, and outcome of 84 patients

\begin{tabular}{|c|c|c|c|c|c|c|}
\hline & \multicolumn{3}{|c|}{$\begin{array}{l}\text { Hematoma Expansion based on } \\
\mathrm{ICH}_{\mathrm{P}}\end{array}$} & \multicolumn{3}{|c|}{$\begin{array}{l}\text { Hematoma Expansion based on } \\
\mathrm{ICH}_{\mathrm{P}+\mathrm{V}}\end{array}$} \\
\hline & $\begin{array}{l}\text { Yes }(37 \\
\text { cases) }\end{array}$ & $\begin{array}{l}\text { No }(47 \\
\text { cases) }\end{array}$ & $P$ value & $\begin{array}{l}\text { Yes }(38 \\
\text { cases) }\end{array}$ & $\begin{array}{l}\text { No }(46 \\
\text { cases) }\end{array}$ & $P$ value \\
\hline Sex & & & 1.00 & & & 0.433 \\
\hline Male/Female & $\begin{array}{l}27 / 10 \\
(73 \% / 27 \%)\end{array}$ & $\begin{array}{l}34 / 13 \\
(72 \% / 28 \%)\end{array}$ & & $\begin{array}{l}26 / 12 \\
(68 \% / \\
32 \%)\end{array}$ & $\begin{array}{l}35 / 11 \\
(76 \% / \\
24 \%)\end{array}$ & \\
\hline Age (years) & $\begin{array}{l}60(50.5 \\
66.5)\end{array}$ & $59(51,67)$ & 0.591 & $\begin{array}{l}57.5(51, \\
66)\end{array}$ & $\begin{array}{l}61(51, \\
67)\end{array}$ & 0.790 \\
\hline $\begin{array}{l}\text { Interval from onset to CT } \\
\text { scan (min) }\end{array}$ & $\begin{array}{l}108(54 \\
225)\end{array}$ & $\begin{array}{l}120(69 \\
206)\end{array}$ & 0.609 & $\begin{array}{l}110(53 \\
224)\end{array}$ & $\begin{array}{l}120(71 \\
207)\end{array}$ & 0.683 \\
\hline $\begin{array}{l}\text { Interval between CT } \\
\text { scans (hour) }\end{array}$ & $\begin{array}{l}7.4(4.2 \\
13.2)\end{array}$ & $\begin{array}{l}12.7(7.7 \\
20.2)\end{array}$ & $0.008 *$ & $\begin{array}{l}7.1(4.3 \\
13.5)\end{array}$ & $\begin{array}{l}12.8(8.0 \\
20.2)\end{array}$ & $0.007 *$ \\
\hline Initial IPH volume (mL) & $\begin{array}{l}18.0(9.8 \\
28.9)\end{array}$ & $\begin{array}{l}15.8(7.3 \\
24.5)\end{array}$ & 0.365 & $\begin{array}{l}17.1 \\
(10.1 \\
28.4)\end{array}$ & $\begin{array}{l}15.6(7.3 \\
25.2)\end{array}$ & 0.388 \\
\hline Initial IVH volume $(\mathrm{mL})$ & $0(0,3.5)$ & $0(0,4.8)$ & 0.244 & $0(0,3.7)$ & $0(0,4.7)$ & 0.261 \\
\hline $\begin{array}{l}\text { Initial IPH + IVH volume } \\
(\mathrm{mL})\end{array}$ & $\begin{array}{l}18.1(10.2 \\
36.1)\end{array}$ & $\begin{array}{l}19.4(12.5 \\
28)\end{array}$ & 0.892 & $\begin{array}{l}19.5 \\
(10.7 \\
35.4)\end{array}$ & $\begin{array}{l}18.9 \\
(12.4 \\
28.7)\end{array}$ & 0.902 \\
\hline IPH volume change (mL) & $\begin{array}{l}37.5(11.3 \\
78.2)\end{array}$ & $\begin{array}{l}0(-1.3 \\
1.5)\end{array}$ & $<0.001 *$ & $\begin{array}{l}35.4 \\
(10.2 \\
77.4)\end{array}$ & $\begin{array}{l}-0.1(-1.3 \\
1.6)\end{array}$ & $<0.001^{*}$ \\
\hline IVH volume change (mL) & $\begin{array}{l}3.8(0 \\
10.8)\end{array}$ & $0(0,0.2)$ & $<0.001 *$ & $\begin{array}{l}3.8(0 \\
10.6)\end{array}$ & $\begin{array}{l}0(0 \\
0.04)\end{array}$ & $<0.001 *$ \\
\hline $\begin{array}{l}\text { IPH + IVH volume } \\
\text { change }(\mathrm{mL})\end{array}$ & $\begin{array}{l}42.5(15.7 \\
84)\end{array}$ & $\begin{array}{l}0.1(-1.5 \\
2.1)\end{array}$ & $<0.001 *$ & $\begin{array}{l}39.7(11 \\
82.1)\end{array}$ & $\begin{array}{l}0(-1.6 \\
1.7)\end{array}$ & $<0.001 *$ \\
\hline IVH at baseline CT scan & $17(45.9 \%)$ & $19(40.4 \%)$ & 0.612 & $\begin{array}{l}17 \\
(44.7 \%)\end{array}$ & $\begin{array}{l}19 \\
(41.3 \%)\end{array}$ & 0.671 \\
\hline $\begin{array}{l}\text { Brain surgery during } \\
\text { hospitalization }\end{array}$ & $23(62.2 \%)$ & $15(31.9 \%)$ & $0.006 *$ & $\begin{array}{l}23 \\
(60.5 \%)\end{array}$ & $\begin{array}{l}15 \\
(32.6 \%)\end{array}$ & $0.011 *$ \\
\hline In-hospital mortality & $15(40.5 \%)$ & $3(6.4 \%)$ & $<0.001 *$ & $\begin{array}{l}15 \\
(39.5 \%)\end{array}$ & $3(6.5 \%)$ & $<0.001 *$ \\
\hline \multicolumn{7}{|c|}{ For continuous variables, median $(25 \%, 75 \%)$ values are reported. } \\
\hline \multicolumn{7}{|c|}{ For number of patients, $\mathrm{N}(\%)$ are reported. } \\
\hline *Statistically significant & ence $(p$ & & & & & \\
\hline
\end{tabular}




\begin{tabular}{|c|c|c|c|c|c|c|}
\hline \multirow[b]{2}{*}{$\begin{array}{l}\text { Poor outcome (death + } \\
\text { mRS }>3 \text { at discharge) }\end{array}$} & \multicolumn{3}{|c|}{$\begin{array}{l}\text { Hematoma Expansion based on } \\
\mathrm{ICH}_{\mathrm{P}}\end{array}$} & \multicolumn{3}{|c|}{$\begin{array}{l}\text { Hematoma Expansion based on } \\
\mathrm{ICH}_{\mathrm{P}+\mathrm{V}}\end{array}$} \\
\hline & $35(94.6 \%)$ & $33(70.2 \%)$ & $0.005^{*}$ & $\begin{array}{l}36 \\
(94.7 \%)\end{array}$ & $\begin{array}{l}32 \\
(69.6 \%)\end{array}$ & $0.004 *$ \\
\hline \multicolumn{7}{|c|}{ For continuous variables, median $(25 \%, 75 \%)$ values are reported. } \\
\hline \multicolumn{7}{|c|}{ For number of patients, $\mathrm{N}(\%)$ are reported. } \\
\hline *Statistically significant & rence $(p<c$ & & & & & \\
\hline
\end{tabular}

When considering the IPH only, a total of 37 patients (44\%) met the criteria of hematoma expansion with an absolute hematoma growth $>6 \mathrm{~mL}$ (31 cases) or relative growth of $>33 \%$ ( 36 cases). The other 47 patients (56\%) did not meet the criteria, thus classified as non- $\mathrm{HE}_{\mathrm{p}}$. Patients with $\mathrm{HE}_{\mathrm{p}}$ had a shorter $\mathrm{CT}$ $\mathrm{F} / \mathrm{U}$ interval (7.4 vs 12.7 hours, $\mathrm{p}=0.008$ ), larger IPH volume change (median $37.5 \mathrm{vs} 0 \mathrm{~mL}, \mathrm{p}<0.001$ ) and IVH volume change (median 3.8 vs $0 \mathrm{~mL}, \mathrm{p}<0.001)$ at $\mathrm{F} / \mathrm{U}$, more brain surgery $(62.2 \%$ vs $31.9 \%, \mathrm{p}=0.006)$. $\mathrm{HE}_{\mathrm{p}}$ patients had a higher in-hospital mortality ( $40.5 \%$ vs $\left.6.4 \%, \mathrm{p}<0.001\right)$, and overall poor outcome with $m R S>3$ at discharge ( 94.6 vs. $70.2 \%, p=0.005)$.

Thirty-six patients (43\%) had IVH at initial presentation. At follow-up, 49 patients exhibited IVH. Among them, there were 15 patients $(15 / 48 ; 31 \%)$ with new IVH (i.e., not initially present at baseline). IVH clot retraction (IVH change $<0 \mathrm{ml}$ ) was observed in 13 patients $(13 / 36 ; 36 \%)$, with two patients exhibiting full resolution of IVH at follow-up. The presence of IVH on the initial CT scan showed no significant difference between patients with or without $\mathrm{HE}_{\mathrm{P}}(45.9 \%$ vs $40.4 \%)$. New IVH (60\% vs $\left.11 \%\right)$, IVH growth $>1 \mathrm{ml}(65 \%$ vs $17 \%)$ and any IVH growth (68\% vs $28 \%)$ were significantly associated with hematoma expansion $(\mathrm{P}<0.001)$. This study is consistent with previous studies $(21-23)$. With respect to the early outcome, IVH at the baseline $\mathrm{CT}$ scan is also associated with mortality and poor functional outcome with crude OR of $4.2(\mathrm{P}=0.013)$ and $4.1(\mathrm{P}=0.061)$, respectively.

\section{Hematoma Expansion Status Defined Using IPH+IVH $\left(\mathrm{HE}_{\mathrm{P}+\mathrm{V}}\right)$}

The expansion results of IPH+IVH are also included in Table 1. When using the same criteria of total volume change of $>6 \mathrm{~mL}$ or relative growth of $>33 \%$ to define the expansion, 38 patients (45\%) were $H E_{P+V}$ and 46 patients (55\%) were non- $H E_{P+V}$. When compared with the $\mathrm{HE}_{\mathrm{P}}$ classification result, there were three crossover cases. One patient with $\mathrm{HE}_{\mathrm{P}}$ was re-classified as non- $\mathrm{HE} \mathrm{E}_{\mathrm{P}+\mathrm{V}}$ (Figure 2a), and two patients with non- $\mathrm{HE}_{\mathrm{P}}$ were re-classified as $\mathrm{HE}_{\mathrm{P}+\mathrm{V}}$ (Figure 2b). All three survived the episode and were discharged from the hospital with $\mathrm{mRS}$ of 4 and 5 . Although the status of three patients was changed, the difference between $\mathrm{HE}_{\mathrm{P}+\mathrm{V}}$ and non-HE $\mathrm{E}_{\mathrm{P}+\mathrm{V}}$ remained the same as those reported between $\mathrm{HE}_{\mathrm{P}}$ and non$\mathrm{HE}_{\mathrm{p}}$, and expanders had higher in-hospital mortality (39.5\% vs $\left.6.5 \%, \mathrm{p}<0.001\right)$, and overall poor outcome (94.7 vs. $69.6 \%, p=0.004)$. 


\section{HE Prediction Performance of Different Radiomics Models}

Three radiomics models were built using the $\mathrm{ICH}$ ROI on the baseline to predict hematoma expansion. The results are summarized in Table 2.

Table 2

The prediction performance of three different radiomics analysis models

\begin{tabular}{|llll|}
\hline & RAP-P & RA $_{\text {PV-P }}$ & RA \\
\hline Accuracy & $0.8(67 / 84)$ & $0.81(68 / 84)$ & $0.86(72 / 84)$ \\
\hline Sensitivity & $0.68(25 / 37)$ & $0.92(34 / 37)$ & $0.82(31 / 38)$ \\
\hline Specificity & $0.89(42 / 47)$ & $0.72(34 / 47)$ & $0.89(41 / 46)$ \\
\hline False Positive Rate & $0.17(5 / 30)$ & $0.28(13 / 47)$ & $0.14(5 / 36)$ \\
\hline False Negative Rate & $0.22(12 / 54)$ & $0.08(3 / 47)$ & $0.15(7 / 48)$ \\
\hline Positive Predictive Value & $0.83(25 / 30)$ & $0.72(34 / 47)$ & $0.86(31 / 36)$ \\
\hline Negative Predictive Value & $0.78(42 / 54)$ & $0.92(34 / 37)$ & $0.85(41 / 48)$ \\
\hline
\end{tabular}

The decision tree of the third model built using the radiomics features of $\mathrm{ICH}_{\mathrm{P}+\mathrm{V}}$ to predict $\mathrm{HE}_{\mathrm{P}+\mathrm{V}}$ is illustrated in Figure 3.

In the first model using the traditional IPH to predict $\mathrm{HE}_{\mathrm{P}}$, i.e. $\mathrm{RA}_{\mathrm{P}-\mathrm{P}}$, there were 25 true positive (TP), 42 true negative (TN), 5 false positive (FP), and 12 false negative (FN) cases. The accuracy, sensitivity and specificity were $80 \%, 68 \%$, and $89 \%$, respectively. When replacing the hematoma ROIs of $\mathrm{ICH}_{\mathrm{P}}$ with $\mathrm{ICH}_{\mathrm{P}+\mathrm{V}}$ for feature selection to predict $\mathrm{HE}_{P}$, i.e. RA $A_{P V-P}$, the accuracy was slightly improved to $81 \%$ with $34 \mathrm{TP}, 34$ $\mathrm{TN}, 13 \mathrm{FP}$, and $3 \mathrm{FN}$ cases. The sensitivity of this prediction model was markedly increased to $92 \%$ with compromised specificity to $72 \%$. When the hematoma ROls of $\mathrm{ICH}_{\mathrm{P}+\mathrm{V}}$ were used to predict $\mathrm{HE}_{\mathrm{P}+\mathrm{V}}$, i.e. $R_{P V-P V}$, the prediction accuracy was further improved to $86 \%$ with $31 \mathrm{TP}, 41 \mathrm{TN}, 5 \mathrm{FP}$, and $7 \mathrm{FN}$ cases. The sensitivity and specificity were $82 \%$ and $89 \%$, respectively. Figure 4 a shows a case example, which is classified as an expander using both criteria. The model built using IPH alone (RAp-P) gives false negative results, but the other two models based on $\mathrm{PPH}+\mathrm{IVH}$ give true positive results and correctly predict this patient as an expander. Figure $4 \mathrm{~b}$ shows another expander case, which is very rare that all three models fail and give false negative results. This patient had a very large hematoma and appeared to be homogeneous on $\mathrm{CT}$, which might be the reason for the false prediction.

\section{Radiologic parameters and early outcome of different decision trees}

The comparison of the radiologic parameters and early outcome between the hematoma expanders and non-expanders of each decision tree was summarized in Table 3. As compared with the 48 non- 
expanders labelled by RAPV-PV, the 36 expanders had significantly shorter CT follow-up intervals, larger hematoma volume change, more brain surgery, higher in-hospital mortality and poor functional outcome at discharge. This finding was consistent with the results of original definition of hematoma expansion (Table 1). On the other hand, there was no significant difference of CT follow-up interval and functional outcome at discharge between 30 expanders and 54 non-expanders classified by RAp-p. It could be attributed to the modest sensitivity $(68 \%)$ of RA $A_{p-P}$ for hematoma expansion. With hematoma ROIs of $\mathrm{ICH}_{\mathrm{P}+\mathrm{V}}$ for feature selection, a total of 47 hematoma expanders tagged by RA $A_{\mathrm{PV}-\mathrm{P}}$ resumed the correlation between hematoma expansion and poor outcome at discharge. However, the CT follow-up interval between the tagged expanders and non-expanders by RA $A_{P V-P}$ showed no significant difference. 
Table 3

Comparison of radiologic parameters and clinical outcome of the labelled patients in different prediction models

\begin{tabular}{|c|c|c|c|}
\hline & \multicolumn{3}{|c|}{ Labelled Hematoma Expansion } \\
\hline & Yes & No & $P$ value \\
\hline$R_{P-P}$ & 30 cases & 54 cases & \\
\hline Median interval from onset to CT scan (mins) & $92(65,167)$ & $137(75,223)$ & 0.637 \\
\hline Median interval between CT scans (mins) & $498(320,924)$ & $706(350,1181)$ & 0.282 \\
\hline Median initial IPH volume (ml) & $16.7(6.7,28.4)$ & $15.9(9,25.2)$ & 0.833 \\
\hline Median initial IVH volume (ml) & $0(0,1.5)$ & $0(0,4.8)$ & 0.100 \\
\hline Median initial IPH + IVH volume (ml) & $18(6.9,30.8)$ & $19.5(12.7,31.5)$ & 0.318 \\
\hline Median IPH volume change (ml) & $25.4(3.4,71.3)$ & $0 . .8(-1.2,4.4)$ & 0.004 \\
\hline Median IVH volume change (ml) & $0.8(0,6.6)$ & $0(0,1.3)$ & 0.014 \\
\hline Median IPH + IVH volume change $(\mathrm{ml})$ & $32(4.6,76)$ & $0.9(-1.3,5.7)$ & $<0.001$ \\
\hline Intraventricular extension & $11(36.7 \%)$ & $25(46.3 \%)$ & 0.393 \\
\hline GCS 3-13 & $12(40 \%)$ & $27(50 \%)$ & 0.379 \\
\hline Brain surgery during hospitalization & $18(60 \%)$ & $20(37 \%)$ & 0.043 \\
\hline In-hospital mortality & $10(33.3 \%)$ & $8(14.8 \%)$ & 0.047 \\
\hline $\mathrm{mRS}$ at discharge $>3$ & $26(86.7 \%)$ & $42(77.8 \%)$ & 0.320 \\
\hline$R A_{P V-P}$ & 47 cases & 37 cases & \\
\hline Median interval from onset to CT scan (mins) & $111(53,167)$ & $147(74,222)$ & 0.961 \\
\hline Median interval between CT scans (mins) & $554(338,1047)$ & $700(335,1213)$ & 0.329 \\
\hline Median initial IPH volume (ml) & $16.0(10.7,25.3)$ & $15.5(6.8,27.6)$ & 0.690 \\
\hline Median initial IVH volume (ml) & $0(0,3.7)$ & $0(0,4.4)$ & 0.987 \\
\hline Median initial IPH + IVH volume (ml) & $20.9(11.3,30.9)$ & $18.5(9.9,32.5)$ & 0.756 \\
\hline Median IPH volume change (ml) & $15.4(2.3,63.2)$ & $0(-1.2,2.1)$ & $<0.001$ \\
\hline Median IVH volume change (ml) & $1.5(0,8.1)$ & $0(-0.1,0.1)$ & 0.002 \\
\hline Median IPH + IVH volume change (ml) & $21.5(3.4,67)$ & $-0.1(-1.5,2.7)$ & $<0.001$ \\
\hline Intraventricular extension & $20(42.6 \%)$ & $16(43.2 \%)$ & 0.949 \\
\hline GCS 3-13 & 18 (38.3\%) & $21(56.8 \%)$ & 0.135 \\
\hline
\end{tabular}




\begin{tabular}{|llll|}
\hline \multicolumn{4}{l}{ Labelled Hematoma Expansion } \\
\hline Brain surgery during hospitalization & $28(59.6 \%)$ & $10(27 \%)$ & $\mathbf{0 . 0 0 3}$ \\
\hline In-hospital mortality & $14(29.8 \%)$ & $4(10.8 \%)$ & $\mathbf{0 . 0 3 5}$ \\
\hline mRS at discharge $>3$ & $42(89.4 \%)$ & $26(70.3 \%)$ & $\mathbf{0 . 0 2 7}$ \\
\hline RA PV-PV & 36 cases & 48 cases & \\
\hline Median interval from onset to CT scan (mins) & $101(51,208)$ & $128(76,210)$ & 0.293 \\
\hline Median interval between CT scans (mins) & $460(269,820)$ & $755(448,1202)$ & $\mathbf{0 . 0 2 8}$ \\
\hline Median initial IPH volume $(\mathrm{ml})$ & $15.8(8.9,26.2)$ & $15.5(7.7,27.1)$ & 0.915 \\
\hline Median initial IVH volume $(\mathrm{ml})$ & $0.1(0,3.9)$ & $0(0,4.1)$ & 0.343 \\
\hline Median initial IPH + IVH volume $(\mathrm{ml})$ & $19.4(11.9,30.3)$ & $18.5(12.5,29.7)$ & 0.574 \\
\hline Median IPH volume change $(\mathrm{ml})$ & $19.2(5,68)$ & $0.3(-1.3,2.9)$ & $<0.001$ \\
\hline Median IVH volume change $(\mathrm{ml})$ & $1.3(0,7.7)$ & $0(0,0.2)$ & $\mathbf{0 . 0 2 1}$ \\
\hline Median IPH + IVH volume change $(\mathrm{ml})$ & $32(5.7,74.6)$ & $0.5(-1.5,4.3)$ & $<0.001$ \\
\hline Intraventricular extension & $18(50 \%)$ & $18(37.5 \%)$ & 0.252 \\
\hline GCS 3-13 & $14(38.9 \%)$ & $25(52.1 \%)$ & 0.230 \\
\hline Brain surgery during hospitalization & $21(58.3 \%)$ & $17(35.4 \%)$ & $\mathbf{0 . 0 3 6}$ \\
\hline In-hospital mortality & $13(36.1 \%)$ & $5(10.4 \%)$ & $\mathbf{0 . 0 0 5}$ \\
\hline mRS at discharge $>3$ & $33(91.7 \%)$ & $35(72.9 \%)$ & $\mathbf{0 . 0 3 0}$ \\
\hline
\end{tabular}

\section{Discussions}

In this study, we investigated the impact of IVH on the radiomic analysis for hematoma expansion by implementation of random forest algorithm using different HE definition and hematoma ROIs of IPH without or with addition of IVH. We compared the prediction performance and clinical outcome correlation of three decision trees ( $R A_{P-P}, R A_{P V-P}$ and $R A_{P V-P V}$ ) in a small case series of $84 \mathrm{SICH}$ patients. Using hematoma ROIs of both IVH and IPH for the revised HE definition and decision tree builtup, $R A_{P V-P V}$ had best prediction performance with preserved clinical outcome correlation of HE. By addition of IVH for feature selection, RA $A_{\mathrm{P}-\mathrm{PV}}$ could improve the sensitivity and resume the outcome correlation of $\mathrm{HE}$ prediction model $\left(\mathrm{RA}_{\mathrm{P}-\mathrm{P}}\right)$ using the traditional approach based on IPH per se.

The predictive indicators for HE reported in the literature included CT angiography spot $\operatorname{sign}(25,29,30)$, NCCT radiological features (hypodensities, blend sign, etc.)(31, 32), and clinical information(25, 30, 32, 33). In recent years, radiomics studies also showed convincing results $(14-17,19)$. The least absolute 
shrinkage and selection operation (LASSO) algorithm was the most applied method for feature selection and model buiding $(14-17,19)$, presumably due to its wide availability. The more sophisticated support vector machine(SVM) algorithm has been applied as well(15). The accuracy, sensitivity, and specificity ranged from 0.64 to $0.88 ; 0.75$ to 0.89 and 0.60 to 0.87 , respectively, which covered a wide range, and were highly dependent on the dataset $(14-17,19)$.

Due to the relatively small sample size, we employed the random forest algorithm to extract imaging features and then implemented a binary decision tree to build the classification model. As a proof of principle study in a small dataset, this approach was more likely to yield satisfactory performance(34). In the conventional analysis using IPH to predict IPH expansion $\left(R_{P}-\mathrm{P}\right)$, our result was comparable to the published studies $(14,17,19)$. Using the combined baseline IPH+IVH to predict the revised $H E_{P+V}$ evaluated by combing both, i.e. the RAPV-PV, it showed the highest accuracy of 0.86 , with the sensitivity of 0.82 and specificity of 0.89 . The results suggest that the additional information of IVH could improve the performance of radiomics analysis for HE prediction. In most patients, the precise separation of IVH and IPH could only be performed manually with subjective judgment based on brain anatomy. The model developed using the combined IPH+IVH can be easily implemented by using an automatic computerbased segmentation tool(35-37). For patients with a high risk of expansion, more aggressive procedures, including immediate surgery, may be considered. Another very helpful clinical application of the HE prediction model is to identify eligible subjects who are likely to show HE to participate in anti-expansion drug trials for $\operatorname{sICH}(13,31)$. For this application, a high specificity is preferred. That is, patients who are unlikely to show HE should not be enrolled, to maximize the power of testing the drug efficacy by using the smallest number of subjects.

The initial presence of IVH was not associated with HE in our study. It was also found in the PREDICT study(25)and the cohort study of the BAT score(31) for ICH expansion prediction. However, IVH had been demonstrated as a risk factor of $\mathrm{HE}$ in a case series of 259 putaminal hemorrhage(38) and the INTERACT study(33). On the other hand, dynamic IVH change, including new IVH (15 cases) and any IVH growth (38 cases) were significantly associated with hematoma expansion in our study $(P<0.001)$. This finding was consistent with previous studies(21-23). With respect to the early outcome, IVH at the baseline CT scan is also associated with mortality and poor functional outcome with crude OR of 4.2 and 4.1, respectively. Considering the impact of IVH on the outcome prediction, and the relationship between the dynamic IVH change and hematoma expansion, addition of IVH ROI into the radiomics analysis might enrich the hematoma feature and strengthen the correlation between clinical outcome and the radiomic prediction model, that was reflected in the improvement of prediction performance of $R A_{P V-P}$ and RA $P V-P V$ compared to RAP-P.

There are several limitations. First, this study was a retrospective design from a single-center with small sample size. Second, the request of the F/U CT scan was at the clinician's discretion, most likely due to the large baseline $\mathrm{ICH}$ and/or worsening symptoms. Consequently, there was a relatively high percentage of patients with hematoma expansion $(37 / 84 ; 44 \%)$, and poor outcomes for almost all expanders (> 
94\%). Third, the radiomics models were built using the random forest algorithm and the decision tree method, without going through cross-validation. Therefore, this should be considered as a pilot study mainly for proof of principle, to demonstrate the feasibility of the analysis based on combined IPH+IVH. In the future, the Al software may be applied to automatically segment IPH and IVH on the baseline and F/U NCCT, to efficiently process a large number of patients and evaluate the clinical role of the developed radiomics prediction models.

\section{Conclusion}

Compared with the conventional radiomics analysis based on IPH per se, addition of IVH into the hematoma ROIs for feature selection and revised HE definition with inclusion of IVH volume change could improve the sensitivity and strengthen the outcome correlation of HE prediction model. These results suggest that the Al software should be applied to segment IPH+IVH in a larger-scale study to develop the radiomics prediction models. A reliable model will not only provide a useful tool to aid in better management for ICH patients, and also to select appropriate patients for enrolling into antiexpansion or neuroprotection drug trials.

\section{Abbreviations}

1. HE: hematoma expansion

2. IPH: intraparenchymal hemorrhage

3. NCCT: noncontrast computed tomography

4. RA: Radiomics analysis

\section{Declarations}

Ethics approval and consent to participate: All procedures performed in studies involving human participants were in accordance with the ethical standards of the institutional and/or national research committee and with the 1964 Helsinki declaration and its later amendments or comparable ethical standards. This retrospective study was approved by the Institutional Review Board of our hospital, ChiMei Medical Center (Date 2018-09-21/ No 10709-010). The requirement to obtain informed consent was waived due to its retrospective nature.

Consent for publication: Not applicable

Availability of data and materials: The complete data are available from the corresponding author on a reasonable request.

Competing interests: The authors declare that they have no conflict of interest.

Funding: No funding was received. 
Author contribution:

Te-Chang Wu: Conceptualization, Data curation, Methodology \& Writing - original draft

Yan-Lin Liu: Investigation, Software, Visualization \& Writing - original draft

Jeon-Hor Chen: Conceptualization, Methodology \& Writing - review \& editing

Yang Zhang: Investigation, Software

Tai-Yuan Chen: Data curation \& Formal analysis

Ching-Chung Ko: Data curation \& Formal analysis

Min-Ying Su: Conceptualization, Methodology \& Writing - review \& editing

Acknowledgments: Not applicable

\section{References}

1. Qureshi Al, Tuhrim S, Broderick JP, Batjer HH, Hondo H, Hanley DF. Spontaneous Intracerebral Hemorrhage. New England Journal of Medicine. 2001;344(19):1450-60.

2. van Asch CJ, Luitse MJ, Rinkel GJ, van der Tweel I, Algra A, Klijn CJ. Incidence, case fatality, and functional outcome of intracerebral haemorrhage over time, according to age, sex, and ethnic origin: a systematic review and meta-analysis. Lancet Neurol. 2010;9(2):167-76.

3. Davis SM, Broderick J, Hennerici M, Brun NC, Diringer MN, Mayer SA, et al. Hematoma growth is a determinant of mortality and poor outcome after intracerebral hemorrhage. Neurology. 2006;66(8):1175-81.

4. Brott T, Broderick J, Kothari R, Barsan W, Tomsick T, Sauerbeck L, et al. Early hemorrhage growth in patients with intracerebral hemorrhage. Stroke. 1997;28(1):1-5.

5. Demchuk AM, Dowlatshahi D, Rodriguez-Luna D, Molina CA, Blas YS, Dzialowski I, et al. Prediction of haematoma growth and outcome in patients with intracerebral haemorrhage using the CTangiography spot sign (PREDICT): a prospective observational study. Lancet Neurol. 2012;11(4):30714.

6. Khosravani H, Mayer SA, Demchuk A, Jahromi BS, Gladstone DJ, Flaherty M, et al. Emergency noninvasive angiography for acute intracerebral hemorrhage. AJNR Am J Neuroradiol. 2013;34(8):1481-7.

7. Dowlatshahi D, Demchuk AM, Flaherty ML, Ali M, Lyden PL, Smith EE. Defining hematoma expansion in intracerebral hemorrhage: relationship with patient outcomes. Neurology. 2011;76(14):1238-44.

8. Barras CD, Tress BM, Christensen S, Collins M, Desmond PM, Skolnick BE, et al. Quantitative CT Densitometry for Predicting Intracerebral Hemorrhage Growth. American Journal of Neuroradiology. 2013;34(6):1139-44. 
9. Boulouis G, Morotti A, Brouwers HB, Charidimou A, Jessel MJ, Auriel E, et al. Association Between Hypodensities Detected by Computed Tomography and Hematoma Expansion in Patients With Intracerebral Hemorrhage. JAMA Neurol. 2016;73(8):961-8.

10. Li Q, Zhang G, Huang YJ, Dong MX, Lv FJ, Wei X, et al. Blend Sign on Computed Tomography: Novel and Reliable Predictor for Early Hematoma Growth in Patients With Intracerebral Hemorrhage. Stroke. 2015;46(8):2119-23.

11. Blacquiere D, Demchuk AM, Al-Hazzaa M, Deshpande A, Petrcich W, Aviv RI, et al. Intracerebral Hematoma Morphologic Appearance on Noncontrast Computed Tomography Predicts Significant Hematoma Expansion. Stroke. 2015;46(11):3111-6.

12. Ng D, Churilov L, Mitchell P, Dowling R, Yan B. The CT swirl sign is associated with hematoma expansion in intracerebral hemorrhage. American Journal of Neuroradiology. 2018;39(2):232-7.

13. Boulouis G, Morotti A, Charidimou A, Dowlatshahi D, Goldstein JN. Noncontrast Computed Tomography Markers of Intracerebral Hemorrhage Expansion. Stroke. 2017;48(4):1120-5.

14. Li H, Xie Y, Wang X, Chen F, Sun J, Jiang X. Radiomics features on non-contrast computed tomography predict early enlargement of spontaneous intracerebral hemorrhage. Clin Neurol Neurosurg. 2019;185:105491.

15. Ma C, Zhang Y, Niyazi T, Wei J, Guocai G, Liu J, et al. Radiomics for predicting hematoma expansion in patients with hypertensive intraparenchymal hematomas. European Journal of Radiology. 2019;115:10-5.

16. Xie H, Ma S, Wang X, Zhang X. Noncontrast computer tomography-based radiomics model for predicting intracerebral hemorrhage expansion: preliminary findings and comparison with conventional radiological model. Eur Radiol. 2020;30(1):87-98.

17. Xu W, Ding Z, Shan Y, Chen W, Feng Z, Pang P, et al. A Nomogram Model of Radiomics and Satellite Sign Number as Imaging Predictor for Intracranial Hematoma Expansion. Front Neurosci. 2020;14:491.

18. Shen Q, Shan Y, Hu Z, Chen W, Yang B, Han J, et al. Quantitative parameters of CT texture analysis as potential markers for early prediction of spontaneous intracranial hemorrhage enlargement. European Radiology. 2018;28(10):4389-96.

19. Chen Q, Zhu D, Liu J, Zhang M, Xu H, Xiang Y, et al. Clinical-radiomics Nomogram for Risk Estimation of Early Hematoma Expansion after Acute Intracerebral Hemorrhage. Academic Radiology. 2021;28(3):307-17.

20. Hemphill JC, 3rd, Bonovich DC, Besmertis L, Manley GT, Johnston SC. The ICH score: a simple, reliable grading scale for intracerebral hemorrhage. Stroke. 2001;32(4):891-7.

21. Steiner T, Diringer MN, Schneider D, Mayer SA, Begtrup K, Broderick J, et al. Dynamics of intraventricular hemorrhage in patients with spontaneous intracerebral hemorrhage: risk factors, clinical impact, and effect of hemostatic therapy with recombinant activated factor VII. Neurosurgery. 2006;59(4):767-73; discussion 73-4. 
22. Yogendrakumar V, Ramsay T, Fergusson D, Demchuk AM, Aviv RI, Rodriguez-Luna D, et al. New and expanding ventricular hemorrhage predicts poor outcome in acute intracerebral hemorrhage. Neurology. 2019;93(9):e879-e88.

23. Yogendrakumar V, Ramsay T, Fergusson DA, Demchuk AM, Aviv RI, Rodriguez-Luna D, et al. Redefining Hematoma Expansion With the Inclusion of Intraventricular Hemorrhage Growth. Stroke. 2020;51(4):1120-7.

24. Broderick JP, Adams HP, Barsan W, Feinberg W, Feldmann E, Grotta J, et al. Guidelines for the Management of Spontaneous Intracerebral Hemorrhage. Stroke. 1999;30(4):905-15.

25. Huynh TJ, Aviv RI, Dowlatshahi D, Gladstone DJ, Laupacis A, Kiss A, et al. Validation of the 9-Point and 24-Point Hematoma Expansion Prediction Scores and Derivation of the PREDICT A/B Scores. Stroke. 2015;46(11):3105-10.

26. Morotti A, Brouwers HB, Romero JM, Jessel MJ, Vashkevich A, Schwab K, et al. Intensive Blood Pressure Reduction and Spot Sign in Intracerebral Hemorrhage: A Secondary Analysis of a Randomized Clinical Trial. JAMA Neurol. 2017;74(8):950-60.

27. Delgado Almandoz JE, Yoo AJ, Stone MJ, Schaefer PW, Goldstein JN, Rosand J, et al. Systematic characterization of the computed tomography angiography spot sign in primary intracerebral hemorrhage identifies patients at highest risk for hematoma expansion: the spot sign score. Stroke. 2009;40(9):2994-3000.

28. Segal M, editor Machine Learning Benchmarks and Random Forest Regression2004.

29. Morotti A, Boulouis G, Charidimou A, Schwab K, Kourkoulis C, Anderson CD, et al. Integration of Computed Tomographic Angiography Spot Sign and Noncontrast Computed Tomographic Hypodensities to Predict Hematoma Expansion. Stroke. 2018;49(9):2067-73.

30. Brouwers HB, Chang Y, Falcone GJ, Cai X, Ayres AM, Battey TW, et al. Predicting hematoma expansion after primary intracerebral hemorrhage. JAMA Neurol. 2014;71(2):158-64.

31. Morotti A, Dowlatshahi D, Boulouis G, Al-Ajlan F, Demchuk AM, Aviv RI, et al. Predicting Intracerebral Hemorrhage Expansion With Noncontrast Computed Tomography. Stroke. 2018;49(5):1163-9.

32. Liu J, Xu H, Chen Q, Zhang T, Sheng W, Huang Q, et al. Prediction of hematoma expansion in spontaneous intracerebral hemorrhage using support vector machine. EBioMedicine. 2019;43:454-9.

33. Wang X, Arima H, Salman RA-S, Woodward M, Heeley E, Stapf C, et al. Clinical Prediction Algorithm (BRAIN) to Determine Risk of Hematoma Growth in Acute Intracerebral Hemorrhage. Stroke. 2015;46(2):376-81.

34. Nasrabadi N. Pattern Recognition and Machine Learning. Journal of Electronic Imaging. 2007;16(4):049901.

35. Yao H, Williamson C, Gryak J, Najarian K. Automated hematoma segmentation and outcome prediction for patients with traumatic brain injury. Artificial Intelligence in Medicine. 2020;107:101910.

36. Patel A, Schreuder FHBM, Klijn CJM, Prokop M, Ginneken Bv, Marquering HA, et al. Intracerebral Haemorrhage Segmentation in Non-Contrast CT. Scientific Reports. 2019;9(1):17858. 
37. Ironside N, Chen C-J, Mutasa S, Sim JL, Marfatia S, Roh D, et al. Fully Automated Segmentation Algorithm for Hematoma Volumetric Analysis in Spontaneous Intracerebral Hemorrhage. Stroke. 2019;50(12):3416-23.

38. Fujii Y, Takeuchi S, Sasaki O, Minakawa T, Tanaka R. Multivariate analysis of predictors of hematoma enlargement in spontaneous intracerebral hemorrhage. Stroke. 1998;29(6):1160-6.

\section{Figures}
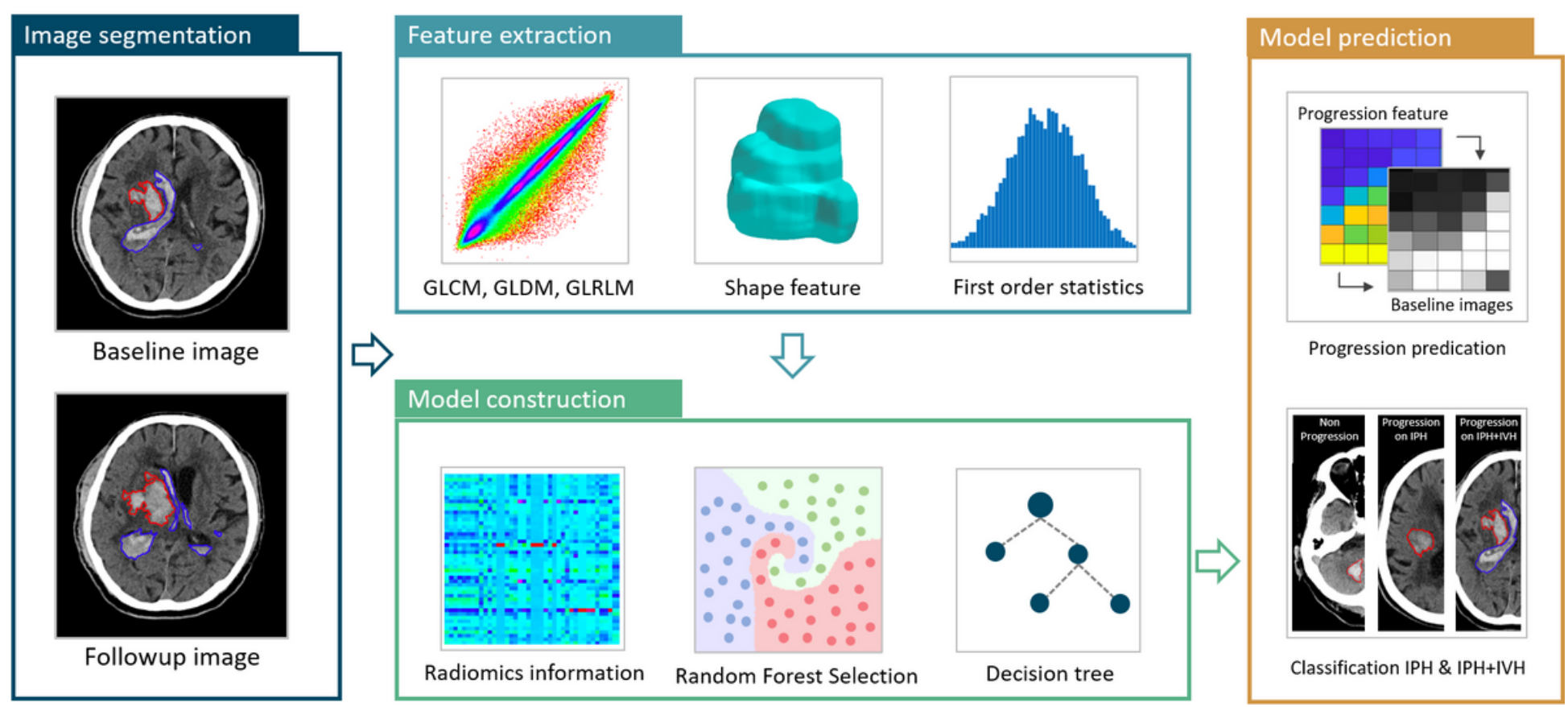

Figure 1

The radiomics analysis flowchart to build the ICH expansion model. The IPH and IVH are segmented by manual tracing of the hematoma on baseline and follow-up CT images. The absolute or percentage volumetric change is calculated to determine whether the patient is an expander, or a non-expander based on IPH or IPH+IVH. The baseline ROI is used to extract radiomics features, and then the important features are selected by using the random forest algorithm to build the prediction model with the decision tree. 


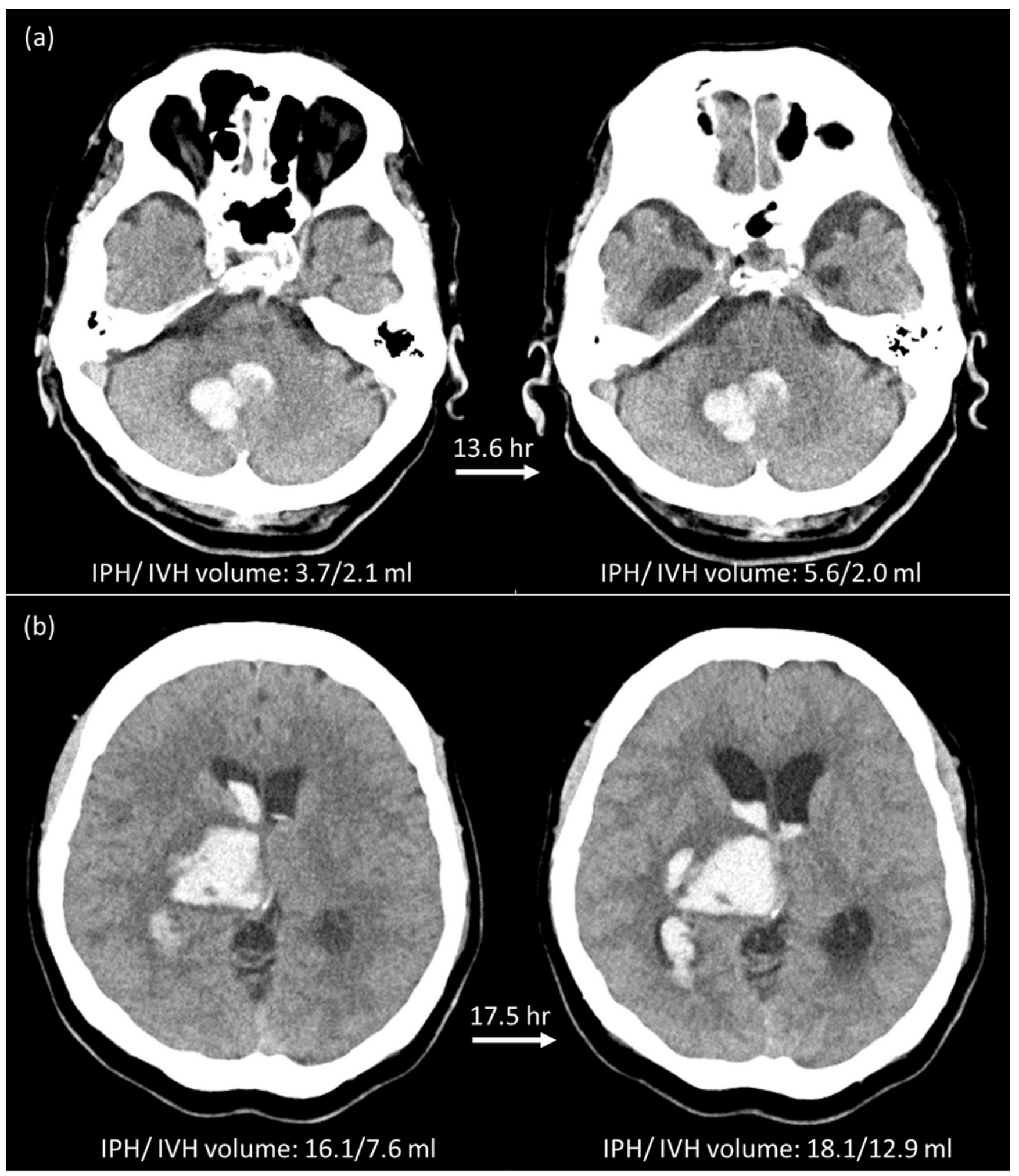

Figure 2

Illustration of two crossover cases. (a) A 94-year-old male with right cerebellar hemorrhage is classified as an expander based on IPH (3.7 to $5.6 \mathrm{ml}, 51 \%$ growth), but is reclassified as a non-expander based on $\mathrm{IPH}+\mathrm{IVH}$ (5.8 to $7.6 \mathrm{ml}, 31 \%$ growth < $33 \%$ threshold). This patient is discharged on Day-74 after ICH with mRS of 5. (b) A 52-year-old female with right thalamic hemorrhage is classified as a non-expander based 
on IPH (16.1 to $18.1 \mathrm{ml})$, but is re-classified as an expander based on IPH+IVH $(23.7$ to $31.0 \mathrm{ml}, 7.3 \mathrm{ml}$ growth $>6 \mathrm{ml}$ threshold). This patient is discharged on Day-70 after ICH with mRS of 5 .

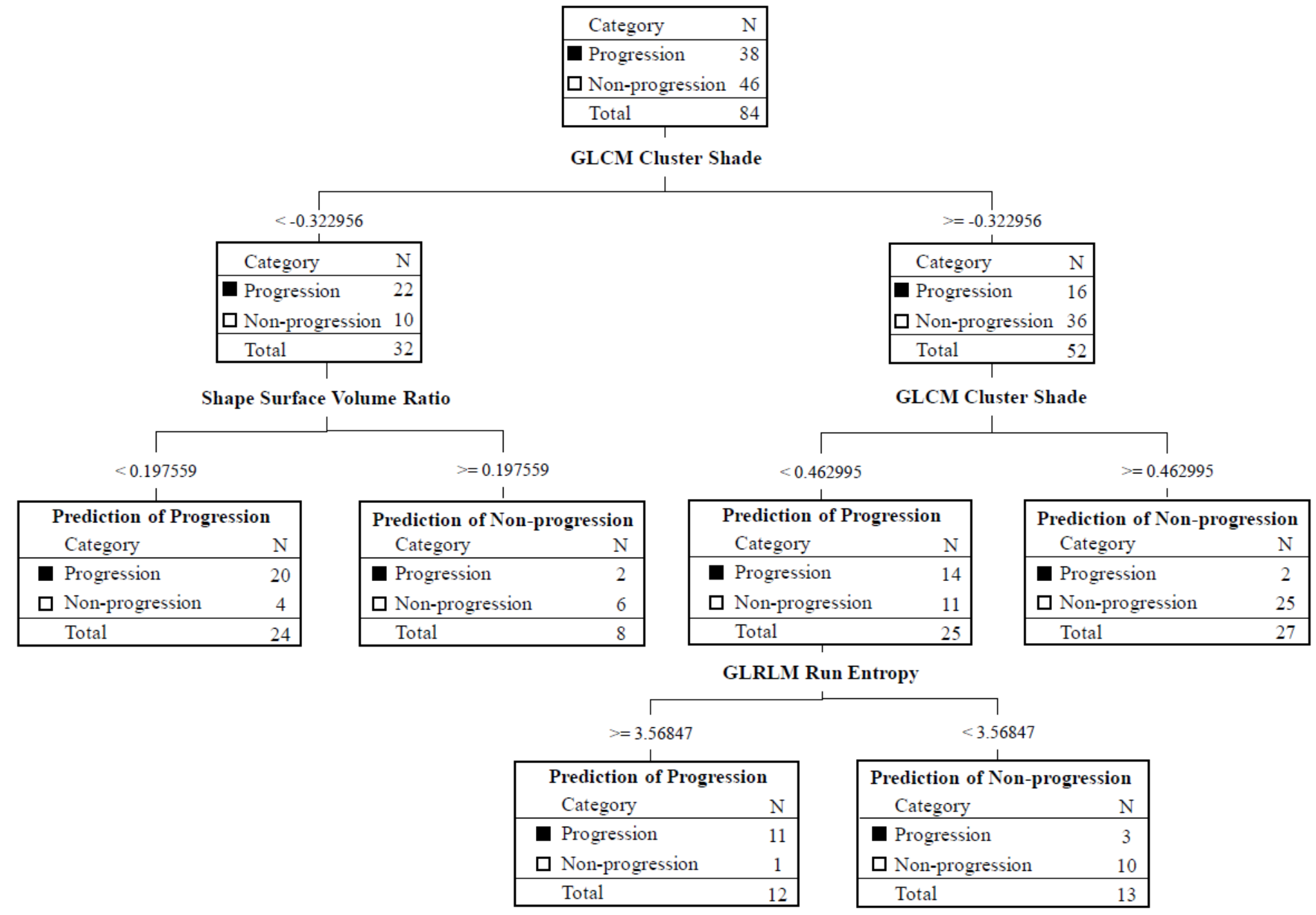

\section{Figure 3}

The decision tree built for the RAPV-PV model, by using radiomics features extracted from IPH+IVH to predict expansion determined based on the IPH+IVH criteria. The final results show 31 true positive, 41 true negative, 5 false positive, 7 false negative, with an overall accuracy of $86 \%$. 

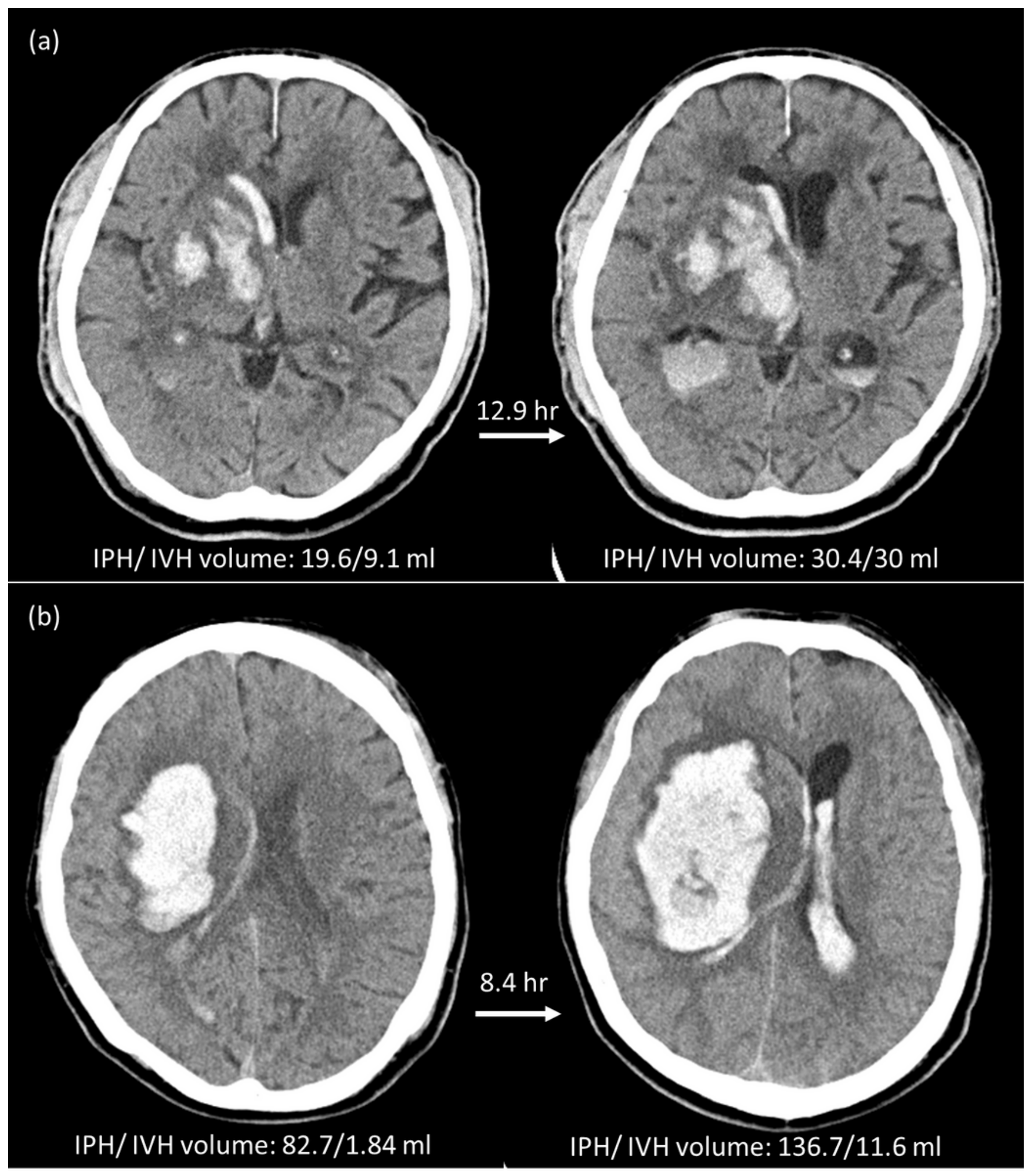

Figure 4

Case illustration of different classification results. (a) A 78-year-old male with right putaminothalamic hemorrhage, classified as an expander either based on IPH or IPH+IVH criteria. This patient is discharged on Day-51 after ICH with mRS of 5. The first radiomics model based on IPH (RAP-P) wrongly predicts this case as a non-expander, but the other two models based on IPH+IVH (RAPV-P, RAPV-PV) correctly predict this case as an expander. (b) A 51-year-old male with right putaminal hemorrhage, classified as an 
expander either based on IPH or IPH+IVH criteria. The patient is discharged on Day- 5 after ICH with mRS of 6 . All three radiomics models give false negative results.

\section{Supplementary Files}

This is a list of supplementary files associated with this preprint. Click to download.

- SupplementaryFiguresBMCNeurology.pdf 